\title{
Coaxial Self-Trapping of White and Gray Regions of an Incandescent Field: A Bright Core with a Dark Cladding
}

\author{
Kailash Kasala and Kalaichelvi Saravanamuttu \\ Department of Chemistry and Chemical Biology, McMaster University, 1280 Main Street West, \\ Hamilton, ON, Canada L8S 4M1 \\ Correspondence should be addressed to Kalaichelvi Saravanamuttu, kalai@mcmaster.ca
}

Received 14 May 2012; Accepted 23 July 2012

Academic Editor: Michael R. Gleeson

Copyright ( $\odot 2012$ K. Kasala and K. Saravanamuttu. This is an open access article distributed under the Creative Commons Attribution License, which permits unrestricted use, distribution, and reproduction in any medium, provided the original work is properly cited.

\begin{abstract}
We report the generation of a self-trapped incoherent hybrid beam comprising a dark-sheathed bright core. The hybrid beam originates from refractive index changes in a photocrosslinkable organosiloxane, which allow simultaneous and cooperative selftrapping of a gray ring with a white core embedded in a broad incandescent beam. The core narrowed and increased in intensity while the encircling gray ring decreased in intensity until rendered very dark. This dark sheath improves light confinement in the bright core and protects it from interactions with nearby self-trapped filaments. This is the first example of a self-trapped hybrid beam, which is moreover spatially and temporally incoherent.
\end{abstract}

\section{Introduction}

In this paper, we report that a new self-trapped spatially and temporally incoherent species—a hybrid beam-forms in a photocrosslinkable organosiloxane $[1,2]$. This photopolymer, which undergoes free-radical polymerization and a corresponding increase in refractive index upon irradiation, can be classified as a self-focusing medium (Figure 1). The hybrid beam forms when an incandescent beam embedded with a gray ring propagates through the photopolymer. Refractive index changes $(\Delta n)$ along the beam path lead to self-trapping of the white core into a narrow, intense bright beam. Simultaneously, the gray ring is depleted of intensity by the self-trapped bright core and self-traps into a very dark sheath. The decreased intensity and lack of divergence of the sheath is the signature of self-trapping dark beams [3].

Our finding is counter-intuitive because it shows for the first time that self-trapped bright and dark regions can coexist within the same optical field. Until now, self-trapped bright and dark beams could only be generated separately under the opposite conditions of self-focusing $[4,5]$ and self-defocusing [3], respectively. For example, self-trapped bright incoherent beams can be generated in a photorefractive crystal under self-focusing conditions, in which $\Delta n$ increases with intensity $[4,5]$. By contrast, self-trapped dark incoherent beams must be generated under self-defocusing conditions [3]. Here, an incoherent beam bearing a dark depression (dip) propagating in an appropriately biased photorefractive crystal induces the greatest $\Delta n$ along the path of the dip. The consequent defocusing of the bright background into the greater index (dark) regions counteracts the divergence of the dip and in this way, generates a selftrapped dark beam [3].

The hybrid beam in our study was created with incandescent light, which is incoherent in space and time. Selftrapping of a wavepacket with such poor correlation of phase and amplitude was experimentally discovered in 1997 [5]. The finding raised fundamental questions about the spatial correlation [6], shape [7], frequency distribution [8], and modulation instability (MI) [9] of incoherent white light. Self-trapping of white light was first demonstrated in a photorefractive crystal, which at very low intensities exhibits a noninstantaneous and saturable photoresponse [5]. These critical properties permit copropagation of the multiple 


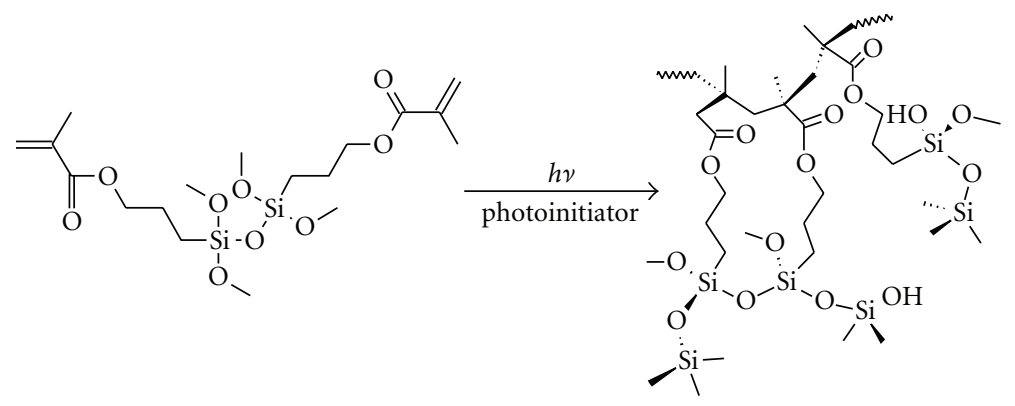

FIGURE 1: Methacrylate substituents in the organosiloxane photopolymer undergo photoinitiated free-radical polymerization. Chain formation leads to a local densification and in turn, an increase in refractive index [1].

modes of white light and time-average out its femtosecond phase fluctuations, respectively.

We have shown that such a saturable and noninstantaneous photoresponse is inherent to the organosiloxane photopolymer [2]. Spatial and temporal variations of $\Delta n$ in the photopolymer can be described through [10]:

$$
\Delta n(r, z, t)=\Delta n_{s}\left\{1-\exp \left[-\frac{1}{U_{0}} \int_{0}^{t-r}|E(t)|^{2} d t\right]\right\}
$$

where $\Delta n_{s}$ is the maximum index change of 1.46 [1] (at saturation), $U_{0}$ is the critical exposure (total energy) required to initiate polymerisation, $\tau$ is the monomer radical lifetime (assumed to be negligible), and $|E(t)|^{2}$ is the square of the electric field amplitude of the incident optical field. We previously reported that the photoresponse of the polymer medium allowed self-trapping of individual incandescent light beams. Studies in this photopolymer also provided insight into the intensity-dependent dynamics, interactions [11], MI [12], and spontaneously formed lattices of selftrapped white beams [13]. We now find that the photopolymer permits simultaneous, coaxial propagation of a bright core and dark sheath as a hybrid beam.

In a typical experiment, a broad, collimated beam of white light (background beam) from a quartz-tungsten-halogen lamp $(\approx 4 \mathrm{~mW})$ was passed through an amplitude mask, which introduced a weakly dark (gray) ring (Figure 2(a)). The ring-embedded background beam was launched into a transparent cuvette containing an organosiloxane photopolymer gel, which was doped with a titanocene photoinitiator $\left(\lambda_{\max }=393 \mathrm{~nm}, 460 \mathrm{~nm}\right)$ to sensitise it to visible light [2]. At the entrance face (propagation distance $(z)=0.0 \mathrm{~mm}$ ), the gray ring had an outer diameter (od) of $350 \mu \mathrm{m}$, while its white core had a FWHM diameter of $220 \mu \mathrm{m}$. The relative intensity of the ring was $44 \%$ while that of the core and background was $50 \%$. The beam profile at $z$ $=6.0 \mathrm{~mm}$ in the photopolymer was imaged with a CCD camera. At early times (when polymerization was negligible), the natural divergence of the background beam causes significant broadening of the ring and core with propagation from $z=0.0 \mathrm{~mm}$ to $z=6.0 \mathrm{~mm}$. Based on the spatial correlation length $\left(l_{c}\right)=0.31 \mu \mathrm{m}$ for the central wavelength $(600 \mathrm{~nm})$ of our incandescent source, the bright core can be calculated using $\theta \approx \lambda / \pi l_{c}^{9}$ to diverge to $\sim 20$-fold its original width at $z=6.00 \mathrm{~mm}$. The decrease in intensity due to the extensive divergence of the core diminished its contrast with the grey ring to the extent that at $z=6.0 \mathrm{~mm}$, the beam appeared effectively uniform with a relative intensity of $\sim 40 \%$ (Figure $2(\mathrm{~b})$ ). This significant divergence, which erases any distinction between dark and bright regions of the optical field, is characteristic of incandescent light and originates from its random and rapid phase fluctuations [14].

Experimental results in Figure 2 show the evolution of the spatial intensity profile of the ring-bearing incandescent beam at $z=6.0 \mathrm{~mm}$. 2D and 3D intensity profiles are shown; for clarity, both a top-down 3D view tracing the intensity increase of the white core and a bottom-up 3D view tracing the depletion of intensity from the gray ring are presented. Over time, polymerization and consequent refractive index changes $(\Delta n)$ along the beam propagation path caused selftrapping of both the gray ring and its white core. At $36 \mathrm{~s}$, self-trapping of the white core was indicated by a decrease in width to $141 \mu \mathrm{m}$ and increase in relative intensity from $40 \%$ to $60 \%$. Simultaneous self-trapping of the gray ring was evidenced through the emergence of a well-defined dark ring with od $=410 \mu \mathrm{m}$. Characteristic of self-trapped dark beams, the gray ring showed a decrease in relative intensity from $40 \%$ to $31 \%$ (Figure 2(c)). This dark- sheathed bright core propagated coaxially without significant divergence as a selftrapped hybrid beam.

Over time, the dark sheath of the hybrid beam continued to darken even as its core increased in intensity. The contrast between the sheath and core maximized at $72 \mathrm{~s}$ at relative intensities of $12 \%$ and $100 \%$, respectively (Figure $2(\mathrm{e})$ ). At this time, the core was only $105 \mu \mathrm{m}$ wide, while the ring od was $440 \mu \mathrm{m}$. Although the core later decreased in intensity with a concomitant (slight) increase in width (characteristic of individual self-trapped white beams) [1], (Figures 2(f) and 2(g)) both the ring and core remained self-trapped. Figure $2(\mathrm{~h})$ shows that at $\sim 1200 \mathrm{~s}$, the widths of the ring and core were $440 \mu \mathrm{m}$ and $90 \mu \mathrm{m}$, respectively. This confirmed that the selftrapped hybrid beam was stable and nontransient.

To explain its mechanism of formation, we first reason that self-trapping of the core of the hybrid beam follows the same process as self-trapping of an individual white beam in the photopolymer [1]. The latter was extensively studied and described elsewhere [1]. Briefly, the core induces 
(a)

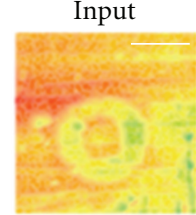

Output

(b)

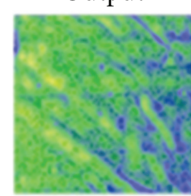

(c)

(d)

(e)
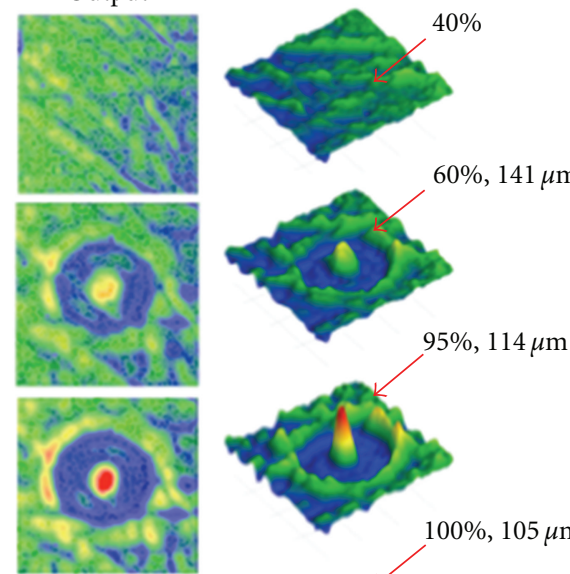

(f)

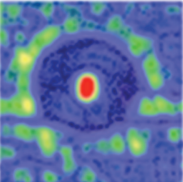

(g)

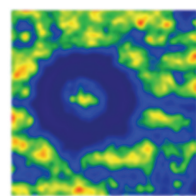

(h)

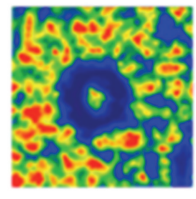

$69 \%, 100 \mu \mathrm{m}$
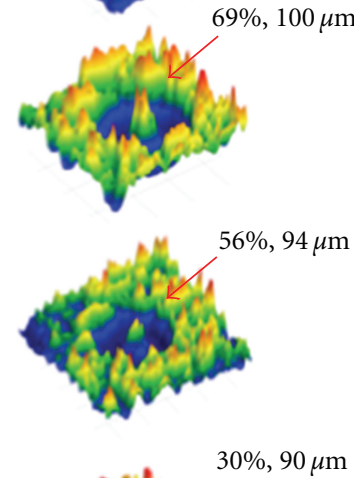
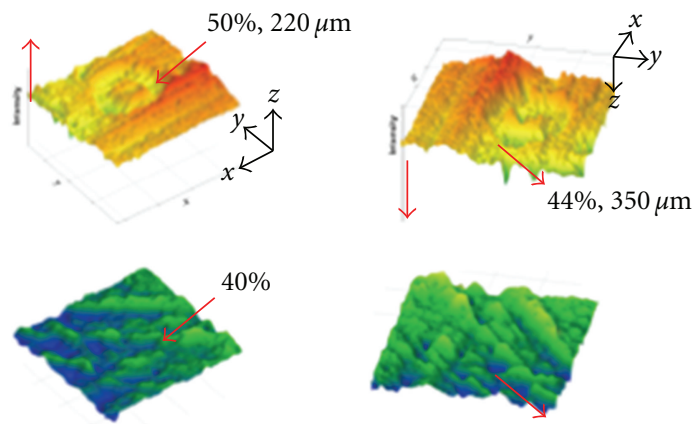

$40 \%$

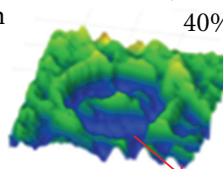

$31 \%, 410 \mu \mathrm{m}$
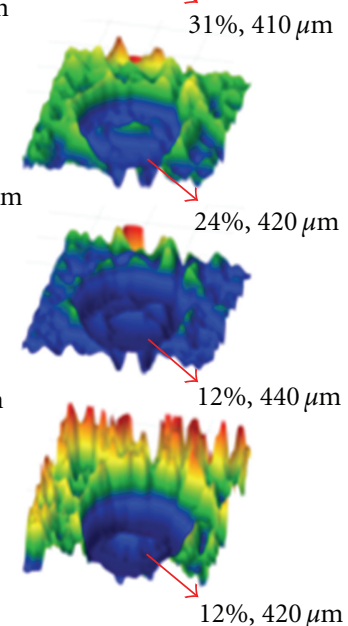

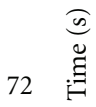

210

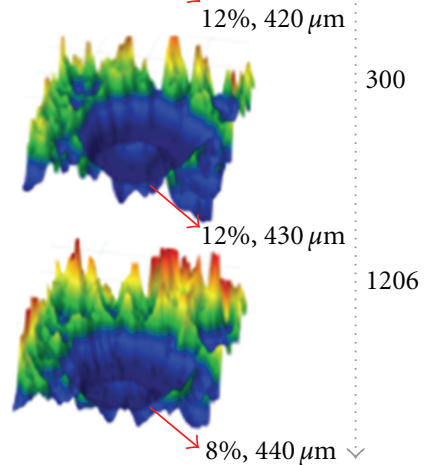

Figure 2: $2 \mathrm{D}$ and 3D spatial intensity profiles of the gray ring-embedded beam at $z=0.0 \mathrm{~mm}$ and (b-f) its evolution at $z=6.0 \mathrm{~mm}$. For clarity, both top-down and bottom-up perspectives of 3D profiles are included. In (a), the scale bar $=250 \mu \mathrm{m}$ and applies to (b-h). The spectral colour scale corresponds to relative intensities of $0 \%$ (blue) to $100 \%$ (red) and is the same in (a-g); for clarity, the scale was magnified by approximately $\times 2$ in $(\mathrm{g})$. Intensities and widths of the ring and core are indicated.

noninstantaneous and saturable refractive index changes along its propagation path. This results in a multimode waveguide, which permits self-consistent propagation and self-trapping of the core as observed in Figure 2. Temporal plots in Figures 3(a) and 3(b) trace the decrease in width and increase in intensity of the self-trapping core. Notably, these plots follow the same trend that was previously observed for an individual self-trapped incandescent beam. This includes the slight increase in width and decrease in intensity at late times when $\Delta n$ of the self-induced waveguide approaches saturation [1]. However, the white core remains self-trapped and never reverts to its original diverged form over long times (>1200s) (Figure 2).

The critical difference in the current study is that the same conditions that cause self-trapping and an increase in intensity of the core elicit self-trapping and a decrease in intensity of the gray ring. To explain this dichotomy, we consider that according to (1), the most intense regions of the optical field (i.e., the bright core and background beam) induce the greatest values of $\Delta n$ in the photopolymer. These bright regions, which have the same initial intensity at $z=0.0 \mathrm{~mm}$, must initiate comparable rates of 

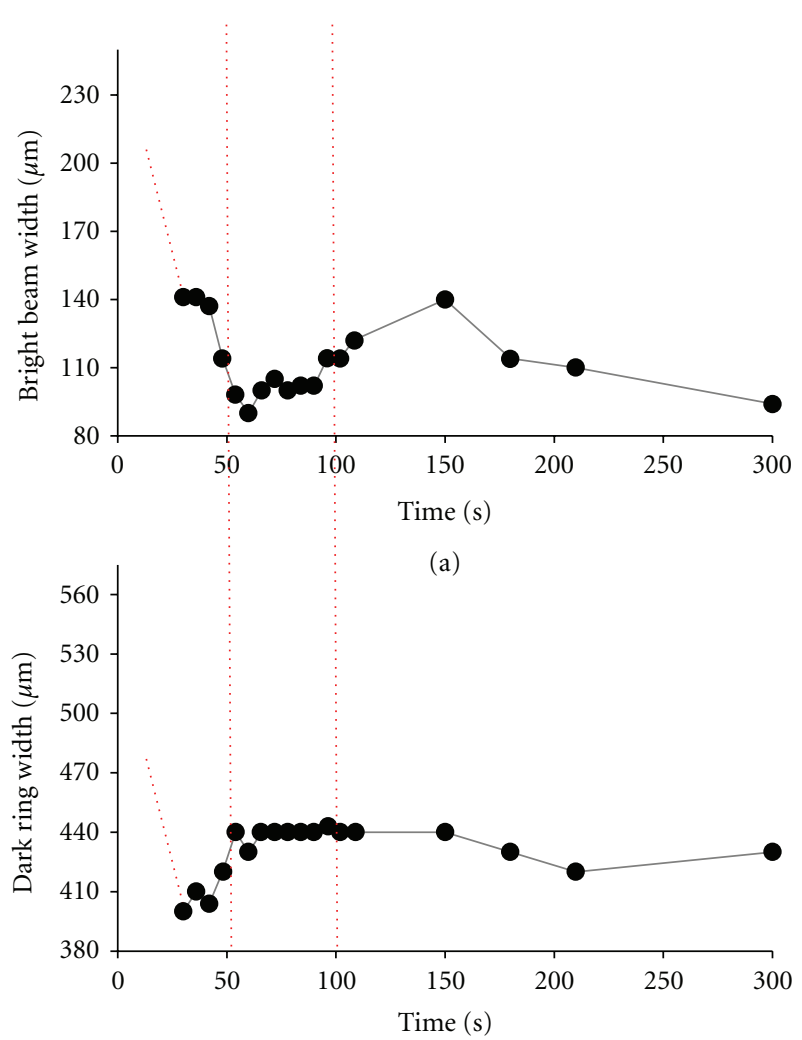

(c)

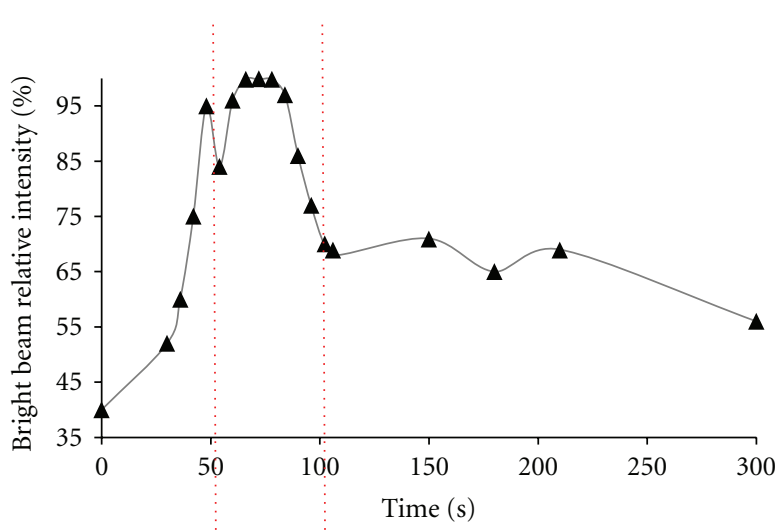

(b)

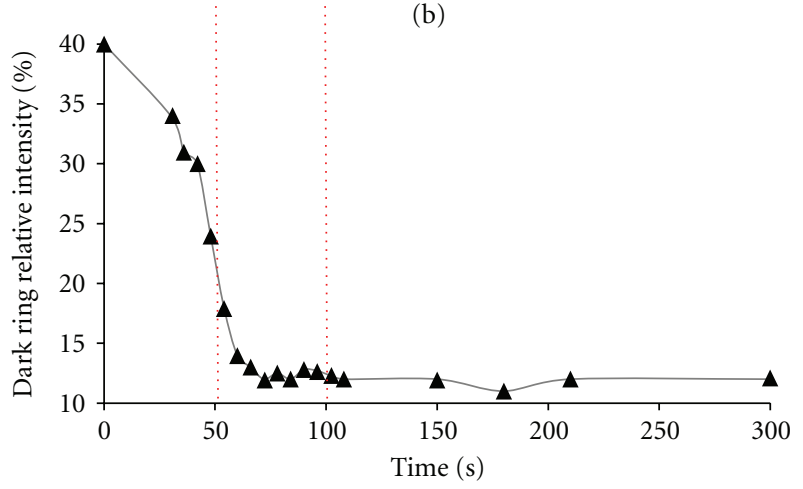

(d)

FIgURE 3: Temporal plots of width and relative intensity of the white core $(\mathrm{a}, \mathrm{b})$ and gray ring $(\mathrm{c}, \mathrm{d})$ of the self-trapped hybrid beam. Dotted lines indicate initial widths of ring and core could not be determined due to the significant divergence of the incandescent beam. Vertical dotted lines are provided as guides to the eye to compare time scales between the different plots.

polymerization. The positive $\Delta n$ values induced in these regions are therefore similar and suppress the divergence of the core and background beam. While this can be directly observed as self-trapping of the core (Figure 2), similar changes to the background beam are not as evident. This is because the background illuminates nearly the entire photopolymer sample and changes to its boundaries cannot be easily detected (vide infra). However, as the core and the background now propagate without divergence, the embedded gray ring is essentially "held" in focus all along the propagation axis. No longer divergent, the ring effectively self-traps and becomes visible at $z=6.0 \mathrm{~mm}$ (Figure 2(c)).

The mechanism described above is confirmed by comparing the temporal variation in the widths of the core and gray ring (Figures $3(\mathrm{a})-3(\mathrm{c})$ ). The plots show that the ring and core self-trap simultaneously within the same time scale: in the first $30 \mathrm{~s}$, the ring and core suffer the greatest decrease in width to $410 \mu \mathrm{m}$ and $141 \mu \mathrm{m}$, respectively. This is consistent with the notion that self-trapping and divergencefree propagation of the bright regions of the optical field causes concomitant self-trapping of the embedded gray ring.

During self-trapping, light intensity spontaneously flows from the gray ring to the core until maximum contrast is achieved between the two. The intensity transfer from the ring to the core is evident in their temporal plots of intensity (Figures 3(b) and 3(d)): at early times, the core increases in intensity from $40 \%$ to $99 \%$ by depleting the ring of intensity during the same period from $40 \%$ to $12 \%$. This results in the simultaneous formation of a self-trapped bright core and self-trapped dark ring (Figure 2). The intensity flow form the ring to the core originates from differences in their photoinduced refractive indices: according to (1), $\Delta n$ in the photopolymer is intensity-dependent. $\Delta n$ induced by the gray ring is therefore smaller than that induced by the white core. The resulting gradient in refractive index between the two regions triggers the flow of intensity from the lowerindex ring to the higher-index core. As its intensity increases, the core induces even greater values of $\Delta n$ and attracts additional intensity from the ring. A cycle is established in which the core continually grows in intensity by depleting the ring, which reaches a minimum intensity of $12 \%$ at $72 \mathrm{~s}$ (Figures $2(\mathrm{e})$ and $3(\mathrm{~d})$ ). Intensity transfer ceases when $\Delta n$ of the core approaches saturation. At this time, similar to individual self-trapped white beams [1], the core intensity decreases together with a slight increase in its width (Figures 2(f) and $3(\mathrm{~b})$ ). While the ring shows a corresponding increase in width, it remains dark and does not undergo any further significant changes (Figures 2(f) and 3(d)).

Our findings indicate that the hybrid beam originates from simultaneous self-trapping and cooperative interactions of the gray ring and white core. Once the hybrid beam is formed, the background beam, which as mentioned 
above also induces index changes, undergoes an entirely different type of nonlinear behaviour, modulation instability (MI) [12]. By $210 \mathrm{~s}$, the uniform background spontaneously divided into multiple filaments (Figure 2(f)). The filaments, which are akin to individual self-trapped beams, were approximately $80 \mu \mathrm{m}$ wide with $\sim 74 \%$ relative intensity. They originate from noise such as random, weak amplitude variations, which become amplified due to polymerization and render the beam unstable. The beam stabilizes by spontaneously dividing into multiple filaments [12]. We note that although they originate in the same medium under identical conditions, MI-induced filaments form at a later time $(210 \mathrm{~s})$ than the self-trapped bright core $(36 \mathrm{~s})$. This is because the dark ring serves as a low-index sheath, which enhances light confinement in the core. This in turn increases the rate and efficiency of self-trapping of the core relative to MI-induced filaments. Furthermore, although filaments immediately surrounding the dark rings were within interacting distance [11] with the self-trapped core (Figure 2(e)), no interactions (e.g., fusion) were observed. We previously showed that interactions between self-trapped white light beams rely on index changes induced by their overlapping optical fields [11]. In the current study, the smaller index of the dark sheath inhibits such overlap and protects the bright core from interactions.

In summary, this to our knowledge is the first report of a self-trapped hybrid beam, which moreover is generated with spatially and temporally incoherent light. The hybrid beam originates from the simultaneous and cooperative selftrapping of a gray ring and its bright core embedded in a broad incandescent beam. The core grows by depleting the ring of most of its intensity and rendering it very dark. Like the cladding of an optical fiber, the dark, low-index sheath enhances light confinement in the core and protects it from interactions. The hybrid beam opens entirely new opportunities to manipulate the confinement and interactions of selftrapped beams through designed introduction of gray and white regions in an optical field. Importantly, they would be accessible with other inexpensive and miniature incoherent light sources such as LEDs. Because of its spatial and temporal incoherence, the hybrid beam also raises intriguing questions about the coherence structure of the dark ring and its bright core.

\section{Acknowledgments}

Funding from Natural Sciences and Engineering Research Council of Canada, Canadian Foundation for Innovation, Ontario Institute of Technology and McMaster University is gratefully acknowledged. The authors thank CIBA for generous donation of IRGACURE-784.

\section{References}

[1] K. Saravanamuttu, X. M. Du, S. I. Najafi, and M. P. Andrews, "Photoinducd structural relaxation and densification in solgel-derived nanocomposite thin films: implications for integrated optics device fabrication," Canadian Journal of Chemistry, vol. 76, no. 11, pp. 1717-1729, 1998.
[2] J. Zhang and K. Saravanamuttu, "The dynamics of selftrapped beams of incoherent white light in a free-radical photopolymerizable medium," Journal of the American Chemical Society, vol. 128, no. 46, pp. 14913-14923, 2006.

[3] Z. Chen, M. Mitchell, M. Segev, T. H. Coskun, and D. N. Christodoulides, "Self-trapping of dark incoherent light beams," Science, vol. 280, no. 5365, pp. 889-891, 1998.

[4] M. Mitchell, Z. Chen, M.-F. Shih, and M. Segev, "Self-trapping of partially spatially incoherent light," Physical Review Letters, vol. 77, no. 3, pp. 490-493, 1996.

[5] M. Mitchell and M. Segev, "Self-trapping of incoherent white light," Nature, vol. 387, no. 6636, pp. 880-883, 1997.

[6] H. Buljan, A. Šiber, M. Soljačić, and M. Segev, "Propagation of incoherent "white" light and modulation instability in noninstantaneous nonlinear media," Physical Review E, vol. 66, no. 3, Article ID 035601, 4 pages, 2002.

[7] H. Buljan, A. Šiber, M. Soljačić, T. Schwartz, M. Segev, and D. N. Christodoulides, "Incoherent white light solitons in logarithmically saturable noninstantaneous nonlinear media," Physical Review E, vol. 68, no. 3, part 2, Article ID 036607, 2003.

[8] H. Buljan, M. Segev, M. Soljačić, N. K. Efremidis, and D. N. Christodoulides, "White-light solitons," Optics Letters, vol. 28, no. 14, pp. 1239-1241, 2003.

[9] T. Schwartz, T. Carmon, H. Buljan, and M. Segev, "Spontaneous pattern formation with incoherent white light," Physical Review Letters, vol. 93, no. 22, Article ID 223901, 2004.

[10] A. S. Kewitsch and A. Yariv, "Self-focusing and self-trapping of optical beams upon photopolymerization," Optics Letters, vol. 21, no. 1, pp. 24-26, 1996.

[11] K. Kasala and K. Saravanamuttu, "An experimental study of the interactions of self-trapped white light beams in a photopolymer," Applied Physics Letters, vol. 93, no. 5, Article ID 051111, 3 pages, 2008.

[12] I. B. Burgess, W. E. Shimmell, and K. Saravanamuttu, "Spontaneous pattern formation due to modulation instability of incoherent white light in a photopolymerizable medium," Journal of the American Chemical Society, vol. 129, no. 15, pp. 4738-4746, 2007.

[13] I. B. Burgess, M. R. Ponte, and K. Saravanamuttu, "Spontaneous formation of 3-D optical and structural lattices from two orthogonal and mutually incoherent beams of white light propagating in a photopolymerisable material," Journal of Materials Chemistry, vol. 18, no. 35, pp. 4133-4139, 2008.

[14] M. Born and E. Wolf, Principles of Optics: Electromagnetic Theory of Propagation, Interference and Diffraction of Light, University of Rochester, New York, NY, USA, 7th edition, 1999. 

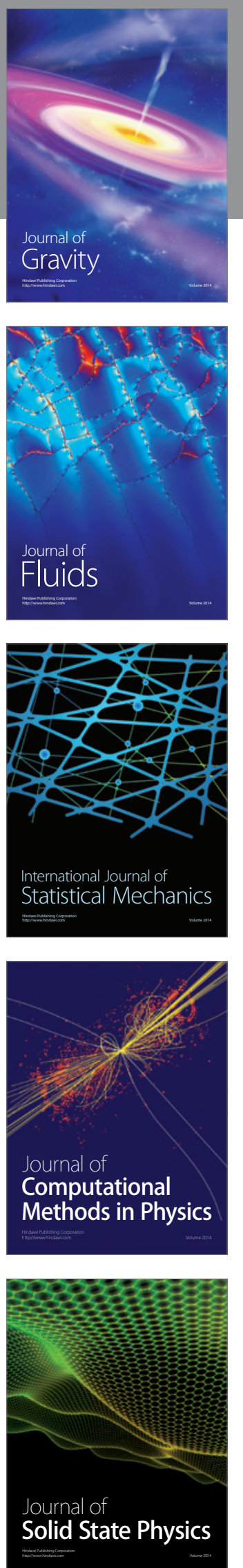

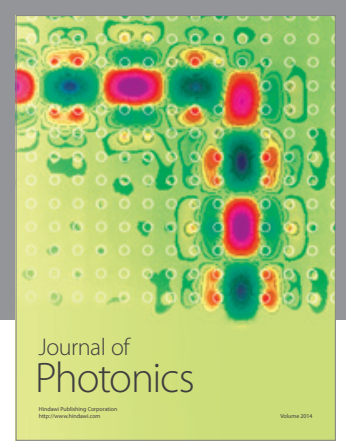

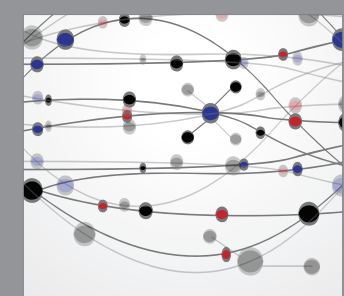

The Scientific World Journal
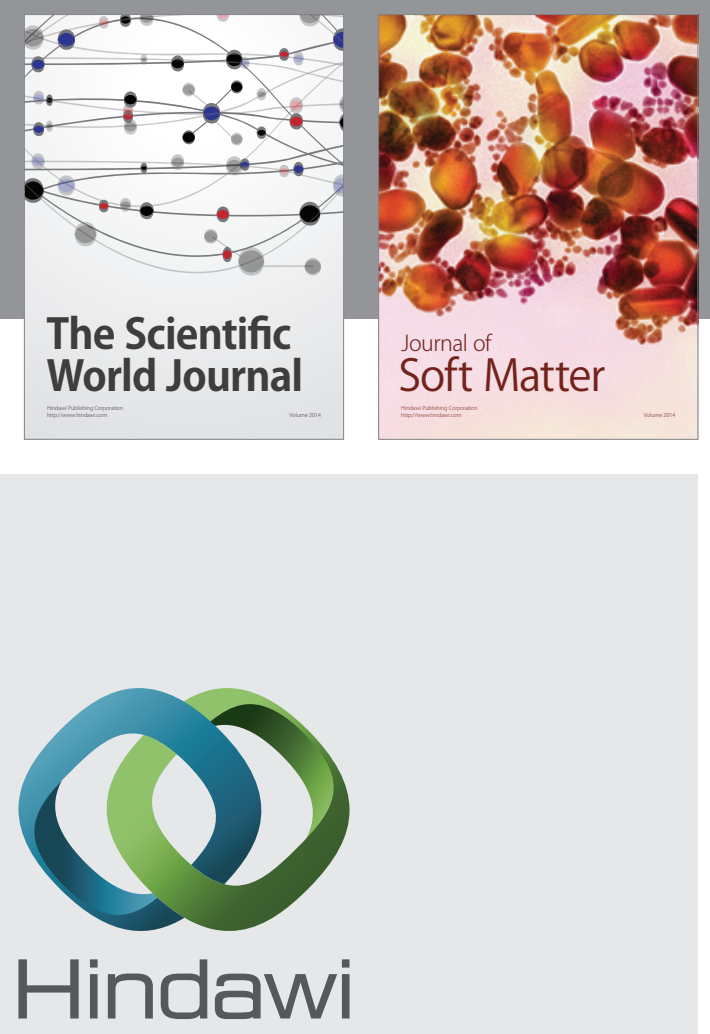

Submit your manuscripts at

http://www.hindawi.com
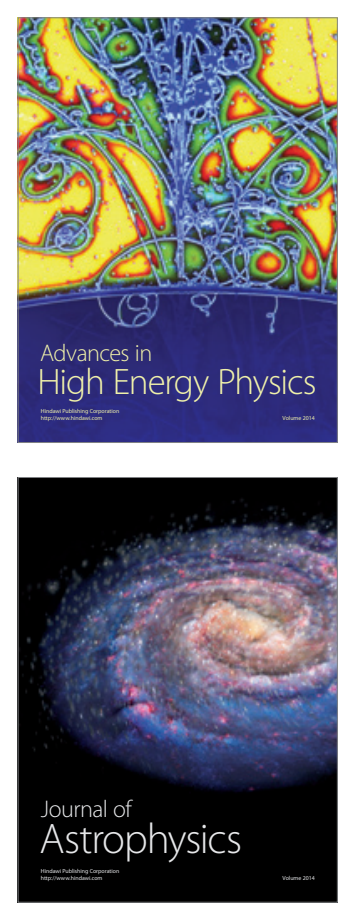
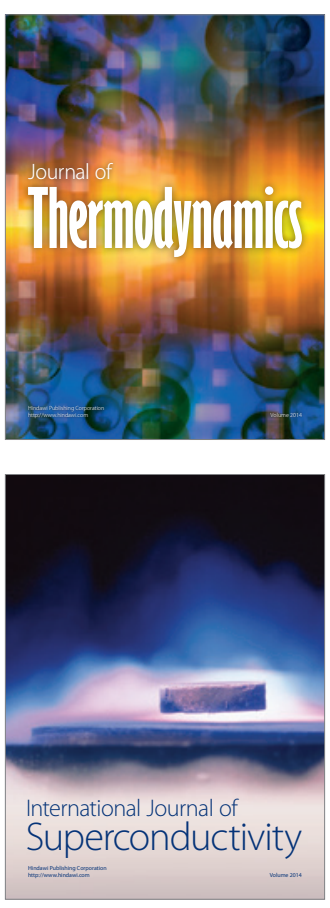
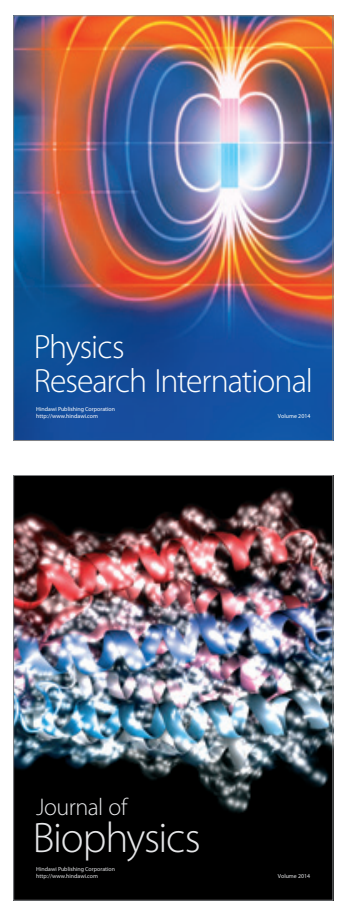
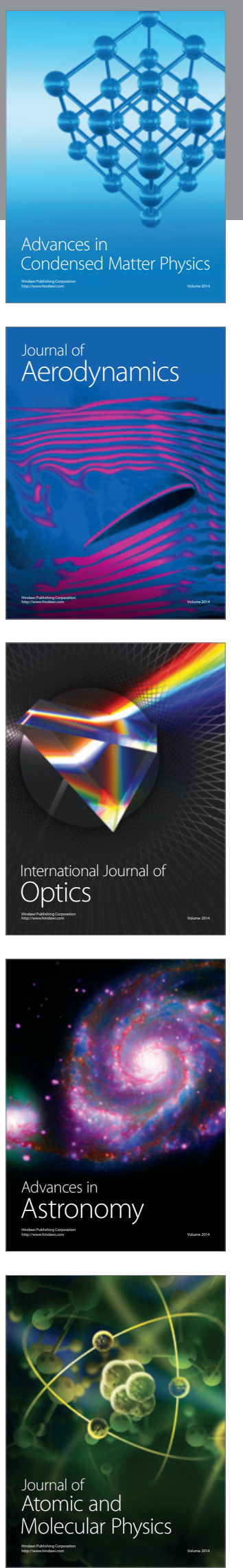